\title{
Synthesis and Performances of Hydrocinnamic Acid Derivatives Containing Alkyl Dihydrazide
}

\author{
Jianxiang $\mathrm{Hu}^{1}$, Lisha $\mathrm{Zhao}^{1}$, Shuangqing $\mathrm{Liu}^{1}$, and Yanhua Cai ${ }^{*}$ \\ ${ }^{1}$ Chongqing Key Laboratory of Environmental Materials \& Remediation Technologies, College of Chemistry and Environmental \\ Engineering, Chongqing University of Arts and Sciences, Chongqing-402160, P.R. China
}

\begin{abstract}
In this work, four hydrocinnamic acid derivatives including $N, N^{\prime}$-succinic bis(hydrocinnamic acid) dihydrazide (HASUD), $N, N^{\prime}$-adipic bis(hydrocinnamic acid) dihydrazide (HAADD), $N, N^{\prime}$-sebacic bis(hydrocinnamic acid) dihydrazide (HASED) and $N, N^{\prime}$-dodecanedioic bis(hydrocinnamic acid) dihydrazide (HADOD) were synthesized via two liquid reactions. And then fourier transform infrared spectrometer and ${ }^{1} \mathrm{H}$ nuclear magnetic resonance were used to characterize molecular structures of four hydrocinnamic acid derivatives. Additionally, the orthogonal experiment obtained the optimum synthesis process of four hydrocinnamic acid derivatives, and the maximum yield of HASUD, HAADD, HASED and HADOD were $33.3 \%, 43.2 \%, 43.5 \%$ and $51.9 \%$, respectively. DMol3 was used to optimize geometric structure and obtain frontier orbital energy. Finally, hydrocinnamic acid derivatives and poly(L-lactide) (PLLA) were blended using the torque rheometer to investigate these role in PLLA resin. The resultant from the melt-crystallization process showed that hydrocinnamic acid derivatives as heterogeneous nucleating agents could significantly enhance PLLA's crystallization.
\end{abstract}

\section{Introduction}

Hydrocinnamic acid as fixative of spice is widely applied in pudding and dairy products, and its derivatives has also obtained more and more attentions due to excellent bioactivity [1]. For example, Wang et al designed and synthesized a series of novel $\alpha$-(phenylmethylene)- $\beta$-oxobenzenepropanoic acid derivatives to evaluate their inhibitory activity against aldose reductase, the related results showed that some derivatives exhibited an inhibitory rate of $80.48 \%$ [2]. For hydrazide compounds, they are not only important organic synthetic raw materials, but also a large number of hydrazide compounds were used in plastics industry to promote polymer's related performances. In order to promote crystallization performance of biodegradable poly(L-lactic acid) (PLLA), A series of compounds having hydrazide groups were synthesized to evaluate their roles in accelerating PLLA's crystallization, and these benzoylhydrazide compounds were proved to be very effective on the enhancement of PLLA's crystallization by research findings, even the crystallization promoting ability is higher than talc [3]. In this study, four hydrocinnamic acid derivatives with different length of methylene chain numbers, derived from hydrocinnamic acid and alkyl dihydrazide, were synthesized through twostep reaction of acylation and amination, and their synthetic processes were investigated by orthogonal experiment, as well as that the optimized geometric structure and frontier orbital energy were obtained by
Dmol3. Additionally, effects of four hydrocinnamic acid derivatives on PLLA's melt-crystallization were also further studied by differential scanning calorimeter (DSC).

\section{Experimental section Results and discussion}

\subsection{Materials and reagents}

4032D PLLA in this study was produced by Nature Works LLC of USA. $N, N^{\prime}$-succinic bis(hydrocinnamic acid) dihydrazide, $N, \quad N^{\prime}$-adipic bis(hydrocinnamic acid) dihydrazide, $N, \quad N$ 'sebacic bis(hydrocinnamic acid) dihydrazide and $N, N^{\prime}$-dodecanedioic bis(hydrocinnamic acid) dihydrazide (labeled as HASUD, HAADD, HASED and HADOD, respectively) were synthesized in our research group, and all reagents used to synthesis aforementioned hydrocinnamic acid derivatives were analytical purity, and these reagents were purchased from Chongqing Huanwei Chemical Company of China.

\subsection{Synthesis of hydrocinnamic acid derivatives}

The synthetic route of hydrocinnamic acid derivatives was shown in Figure.1. Firstly, the hydrocinnamoyl chloride was prepared through the acylation reaction of hydrocinnamic acid. And then the hydrocinnamoyl chloride was added into the mixed solution including alkyl dihydrazide and $N, N$-dimethylformamide, and the mixed solution was stirred under ice bath for a period of time,

"Corresponding author: caiyh651@aliyun.com 
heated up to $60^{\circ} \mathrm{C}$ for further reaction. Finally, the mixed solution was poured into the water and filtrated, followed by drying at $35^{\circ} \mathrm{C}$ in vacuum.

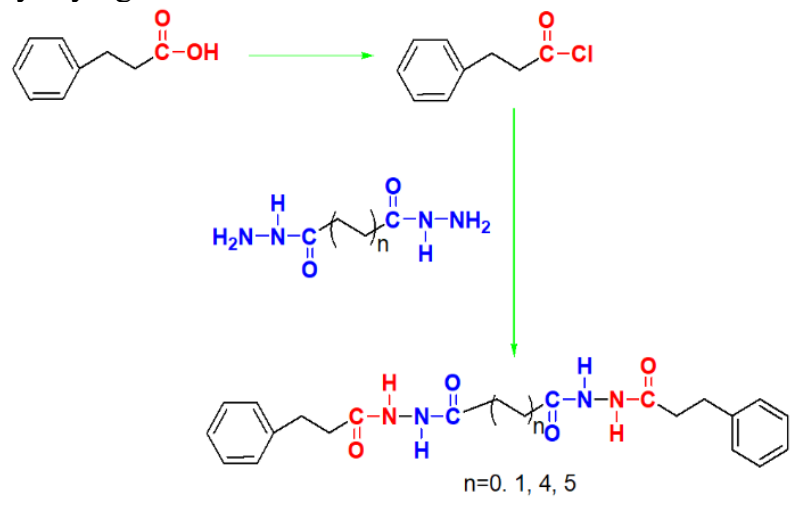

Fig. 1. Synthetic route of four hydrocinnamic acid derivatives

\subsection{Preparation of modified PLLA samples}

The blend of PLLA with $2 \mathrm{wt} \%$ HASUD, 2 wt $\%$ HASED and $2 \mathrm{wt} \%$ HADOD were carried out in a torque rheometer, and the blending operation was similar with our previous works [4-7]. Typical blend procedure of modified PLLA was as follows: PLLA and hydrocinnamic acid derivative were mixed at $190{ }^{\circ} \mathrm{C}$, and the initial rotation speed was set at $32 \mathrm{rpm}$ for $7 \mathrm{~min}$, then $64 \mathrm{rpm}$ for $10 \mathrm{~min}$. Then the mixture were hot pressed at $190{ }^{\circ} \mathrm{C}$ under $20 \mathrm{MPa}$ for 5 $\mathrm{min}$, and further cool pressed at room temperature under $20 \mathrm{MPa}$ for $10 \mathrm{~min}$ to prepare modified PLLA samples with $0.4 \mathrm{~mm}$ thickness for the following measurement about the melt-crystallization.

\subsection{Test and characterization}

The molecular structures of four hydrocinnamic acid derivatives were determined by Fourier Transform Infrared Spectrometer (FT-IR) and ${ }^{1} \mathrm{H}$ Nuclear Magnetic Resonance $\left({ }^{1} \mathrm{H} \mathrm{NMR}\right)$. And the $\mathrm{KBr}$ pellet was used to prepare FT-IR testing sample of hydrocinnamic acid derivative, the wavenumber was from $4000 \mathrm{~cm}^{-1}$ to 400 $\mathrm{cm}^{-1}$; the deuteration DMSO was used to dissolve four hydrocinnamic acid derivatives for ${ }^{1} \mathrm{H}$ NMR measurement. The melt-crystallization processes from the melt $\left(190^{\circ} \mathrm{C}\right)$ of all testing samples, including the PLLA modified by four hydrocinnamic acid derivatives and neat PLLA, were recorded by Q2000 DSC with $50 \mathrm{~mL} / \mathrm{min}$ nitrogen. And the modified PLLA samples of PLLA/2\%HASUD, PLLA $/ 2 \%$ HASED and PLLA/2\%HADOD and neat PLLA were eliminated heat history to ensure the same testing level.

\section{Results and discussion}

\subsection{Molecular structures}

The molecular structures of four hydrocinnamic acid derivatives were characterized by FT-IR and ${ }^{1} \mathrm{H} N \mathrm{NMR}$, and the relevant data are follow:

HASUD: FT-IR(KBr) $\quad$ v: $3446.9 ， 3216.4 ， 3044.4$
$1662.8,1596.9,1482.4,1450.9,1419.9,1321.3,1191.2$, $1165.4,1071.1,1024.3,933.9,741.5,698.2,573.7 \mathrm{~cm}^{-1}$; ${ }^{1} \mathrm{H}$ NMR (DMSO, $\left.400 \mathrm{MHz}\right) \delta$ : ppm; 9.80 (s, 1H, NH), $9.78(\mathrm{~s}, 1 \mathrm{H}, \mathrm{NH}), 7.16 \sim 7.29(\mathrm{~m}, 5 \mathrm{H}, \mathrm{Ar}), 2.80 \sim 2.84(\mathrm{t}$, $\left.4 \mathrm{H}, \mathrm{CH}_{2}\right), 2.36 \sim 2.44\left(\mathrm{~m}, 2 \mathrm{H}, \mathrm{CH}_{2}\right)$.

HAADD: FT-IR(KBr) v: 3448.4, 3219.9, 3028.5, 2941.5, 2868.1, 1658.6, 1600.8, 1478.6, 1412.3, 1260.6, 1221.3, 1190.9, 1074.2, 1031.3, 930.9, 739.2, 698.6, 667.9, 563.4, $523.5 \mathrm{~cm}^{-1}$; ${ }^{1} \mathrm{H}$ NMR (DMSO, $\left.400 \mathrm{MHz}\right) \delta$ : ppm; $9.75(\mathrm{~s}, 1 \mathrm{H}, \mathrm{NH}), 9.70(\mathrm{~s}, 1 \mathrm{H}, \mathrm{NH}), 7.16 \sim 7.27(\mathrm{~m}, 5 \mathrm{H}$, Ar), $2.78 \sim 2.84\left(\mathrm{t}, 4 \mathrm{H}, \mathrm{CH}_{2}\right), 2.40 \sim 2.49\left(\mathrm{~m}, 2 \mathrm{H}, \mathrm{CH}_{2}\right)$, $1.50\left(\mathrm{~s}, 2 \mathrm{H}, \mathrm{CH}_{2}\right)$.

HASED: FT-IR(KBr) v: 3448.0，3315.8，3216.3, 3029.2, 2924.0, 2850.4, 1633.4, 1600.7, 1530.3, 1483.5, $1450.9,1414.5,1377.3,1219.4,1188.7,1159.4,1071.1$, 1029.9, 933.0, 750.3, 720.9, 698.2 $\mathrm{cm}^{-1} ;{ }^{1} \mathrm{H}$ NMR (DMSO, $400 \mathrm{MHz}) \delta$ : ppm; $9.72(\mathrm{~s}, 1 \mathrm{H}, \mathrm{NH}), 9.67$ (s, 1H, NH), $7.09 \sim 7.33(\mathrm{~m}, 5 \mathrm{H}, \mathrm{Ar}), 2.81 \sim 2.84\left(\mathrm{t}, 4 \mathrm{H}, \mathrm{CH}_{2}\right), 2.40 \sim$ $2.44\left(\mathrm{t}, 2 \mathrm{H}, \mathrm{CH}_{2}\right), 1.18 \sim 1.25\left(\mathrm{~m}, 6 \mathrm{H}, \mathrm{CH}_{2}\right)$.

HADOD: FT-IR (KBr) v: 3316.7, 3217.4, 2921.7, 2849.7, 1633.0, 1600.5, 1535.7, 1483.2, 1413.3, 1379.9, $1338.8,1270.9,1213.6,1162.3,1011.9,973.7,932.8$, $697.7 \mathrm{~cm}^{-1}$; ${ }^{1} \mathrm{H}$ NMR (DMSO, $\left.400 \mathrm{MHz}\right) \delta$ : ppm; 9.72 (s, $1 \mathrm{H}, \mathrm{NH}), 9.67(\mathrm{~s}, 1 \mathrm{H}, \mathrm{NH}), 7.17 \sim 7.30$ (m, 5H, Ar), $2.80 \sim 2.84\left(\mathrm{t}, 4 \mathrm{H}, \mathrm{CH}_{2}\right), 2.40 \sim 2.50\left(\mathrm{t}, 2 \mathrm{H}, \mathrm{CH}_{2}\right), 1.98 \sim$ $2.11\left(\mathrm{~m}, 4 \mathrm{H}, \mathrm{CH}_{2}\right), 1.22 \sim 1.24\left(\mathrm{~m}, 4 \mathrm{H}, \mathrm{CH}_{2}\right)$.

Through analysis of FT-IR and ${ }^{1} \mathrm{H} \quad$ NMR characterization data, four hydrocinnamic acid derivatives were successfully synthesized via acylation reaction and amination reaction.

\subsection{Synthetic process and geometry optimization}

The synthetic processes were studied by orthogonal experiment, three factors included mole ratio, reaction time and reaction temperature. And the optimized synthetic processes was shown in Table.1.

Table1. Optimized synthetic processes of four hydrocinnamic acid derivatives

\begin{tabular}{ccccc}
\hline Compound & $\begin{array}{c}\text { Mole ratio } \\
\mathbf{a}\end{array}$ & $\begin{array}{c}\text { time/ } \\
\mathbf{h}\end{array}$ & $\begin{array}{c}\text { temperature/ } \\
{ }_{\mathbf{0}} \mathbf{C}\end{array}$ & $\begin{array}{c}\text { Yield/ } \\
\mathbf{\%}\end{array}$ \\
\hline HASUD & $5: 2$ & 5 & 40 & 33.3 \\
HAADD & $9: 4$ & 4 & 40 & 43.2 \\
HASED & $5: 2$ & 5 & 50 & 43.5 \\
HADOD & $9: 4$ & 6 & 40 & 51.9 \\
\hline a: hydrocinnamoyl chloride: alkyl dihydrazide &
\end{tabular}

The geometric structure was optimized via DMol3 calculation, and the optimized geometric structures of four hydrocinnamic acid derivatives were displayed in Figure.2. Meantime, the frontier orbital energy including HOMO and LUMO were also obtained after geometry optimization. Figure. 3 is HOMO and LUMO of four hydrocinnamic acid derivatives. As seen in Fig.3, Both HOMO and LUMO focus on amide group, but LUMO is more dispersed comparing with HOMO. And the HOMO and LUMO are $-0.2097 \mathrm{eV}$ and $-0.0569 \mathrm{eV}$ for HASUD, - 
$0.2021 \mathrm{eV}$ and $-0.0563 \mathrm{eV}$ for HAADD, $-0.2034 \mathrm{eV}$ and $0.0522 \mathrm{eV}$ for HASED, $-0.2016 \mathrm{eV}$ and $-0.0498 \mathrm{eV}$ for HADOD, respectively. Through analysis HOMO and LUMO data, it is found that, apart from the HAADD, the HOMO decreases with increasing the alkyl, but the LUMO regularly decreases.
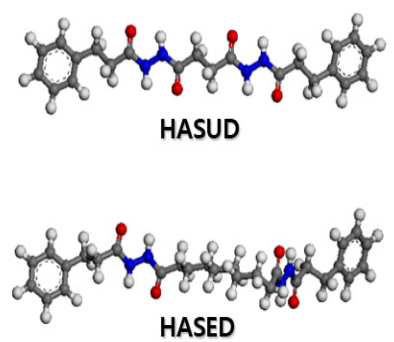
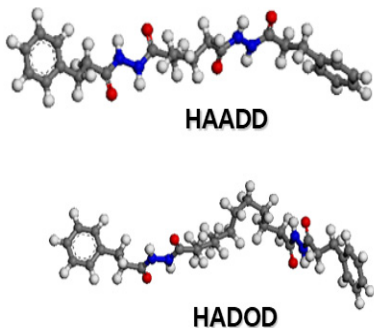

Fig. 2. Optimized geometric structures of HASUD, HAADD, HASED and HADOD

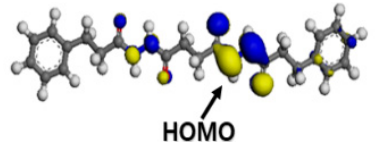

HOMO
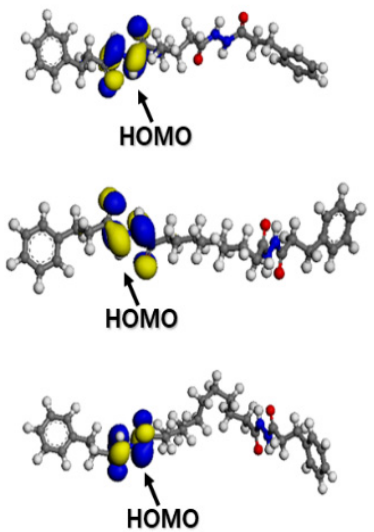

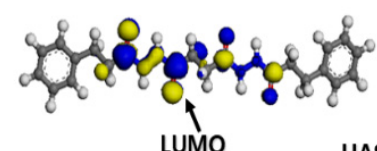

HASUD

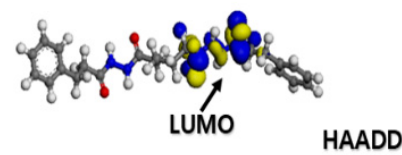

HAADD

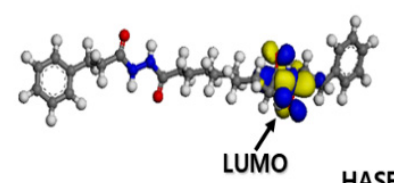

HASED

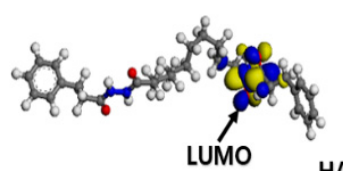

HADOD
Fig. 3. HOMO and LUMO of HASUD, HAADD, HASED and HADOD

\subsection{Nucleation effect of hydrocinnamic acid derivatives}

The synthesized hydrocinnamic acid derivatives were used to evaluate their roles in melt-crystallization process of PLLA. Figure. 4 is the melt-crystallization process of the neat PLLA and PLLA containing three hydrocinnamic acid derivatives at a cooling rate of $1{ }^{\circ} \mathrm{C} / \mathrm{min}$, and the relevant DSC curves and data were recorded by Q2000 DSC. Compared to the DSC curve of the neat PLLA, PLLA/2\%HASUD, PLLA/2\%HASED and PLLA/2\%HADOD samples have very obvious and sharp melt-crystallization peak in DSC curves, indicating that the addition of three hydrocinnamic acid derivatives of HASUD, HASED and HADOD promotes the meltcrystallization of PLLA, the further reason may be that these hydrocinnamic acid derivatives supply enough nucleus in matrix to lead to the fast nucleation rate, and promote the crystallization to occur at a high temperature. Furthermore, among three modified PLLA samples, the PLLA/2\%HASUD sample exhibits the highest melt- crystallization peak temperature of $129.8{ }^{\circ} \mathrm{C}$ and the largest melt-crystallization enthalpy of $49.1 \mathrm{~J} / \mathrm{g}$, as well as the sharpest melt-crystallization peak, showing that the HASUD plays the best promoting role in PLLA's crystallization in this work.

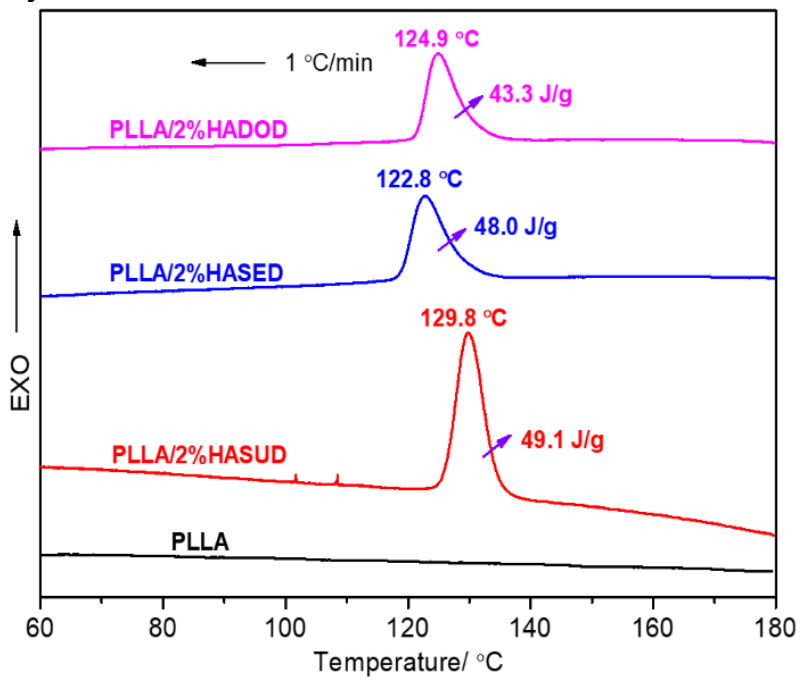

Fig. 4. Melt-crystallization DSC curves of the neat PLLA and PLLA containing three hydrocinnamic acid derivatives

\section{Conclusions}

Acylation and amination reactions were used to synthesis four hydrocinnamic acid derivatives HASUD, HAADD, HASED and HADOD, and the molecular structures of these synthetic compounds were proved by the FT-IR and ${ }^{1} \mathrm{H}$ NMR. The optimized synthetic process obtained through the orthogonal experiment were as follow: for HASUD, the molar ratio $5: 2$, reaction temperature $40{ }^{\circ} \mathrm{C}$, reaction time $5 \mathrm{~h}$, the yield was $33.3 \%$; for HAADD, the molar ratio $9: 4$, reaction temperature $40{ }^{\circ} \mathrm{C}$, reaction time $4 \mathrm{~h}$, the yield was $43.2 \%$; for HASED, the molar ratio 5:2, reaction temperature $50{ }^{\circ} \mathrm{C}$, reaction time $5 \mathrm{~h}$, the yield was $43.5 \%$; for HADOD, the molar ratio $9: 4$, reaction temperature $40{ }^{\circ} \mathrm{C}$, reaction time $6 \mathrm{~h}$, the yield was $51.9 \%$. The DMol3 calculation obtained the optimization geometric structures of four hydrocinnamic acid derivatives and their corresponding frontier orbital energy. Overall, the HOMO and LUMO almost decreased with increasing of alkyl. The melt-crystallization processes of PLLA with ydrocinnamic acid derivatives showed that the hydrocinnamic acid derivatives could significantly accelerate the PLLA's crystallization in cooling, and the reason is that these hydrocinnamic acid derivatives played a role of nucleating agents for promoting PLLA's crystallization. Among these modified PLLA with hydrocinnamic acid derivatives, the PLLA/2\% HASUD sample had the highest melt-crystallization peak temperature in the DSC curve and the largest meltcrystallization enthalpy.

\section{Acknowledgements}

This work was supported by Foundation of Chongqing Municipal Science and Technology Commission 
(cstc2019jcyj-msxmX0876) and Scientific and Technological Research Program of Chongqing Municipal Education Commission (project number KJQN201801319).

\section{References}

1. F.X. Niu, Y.P. Du, Y.B. Huang, H.T. Zhou, J.Z. Liu, Synthetic Biology Journal, 337, 1(2020)

2. S.J. Wang, Z.Y. Zhang, J. Wu, W.F. Zhou, M.S. Cheng, Chinese Journal of Medicinal Chemistry, 5, 16(2006)

3. N. Kawamoto, A. Sakai, T. Horikoshi, T. Urushihara, E. Tobita, Journal of Applied Polymer Science,198, 103(2007)

4. L.S. Zhao, Y.H. Cai, H.L. Liu, E-Polymers, 141, 19(2019)

5. L.S. Zhao, Y.H. Cai, Polymer Science, Series A, 777, 60(2018)

6. H. Huang, Y.H. Zhang, L.S. Zhao, G.M. Luo, Y.H. Cai, Materiale Plastice, 28, 57(2020)

7. Y.H. Cai, L.S. Zhao, Polymer Bulletin, 2295, 76(2019) 